\title{
Avaliação antropométrica e análise do consumo alimentar de universitários da Faculdade de Ciências Aplicadas - UNICAMP
}

\author{
Mayara Lilian Paulino Miranda ${ }^{1}$, Mayra Mayumi Kamiji ${ }^{2}$, Cristiano Torezzan ${ }^{3}$, Adriane \\ Elisabete Costa Antunes ${ }^{4}$
}

\begin{abstract}
Somadas a situações próprias da adolescência, mudanças oriundas do ingresso no meio universitário, como modificações no padrão alimentar, na prática de atividade física e no consumo de álcool e cigarros podem interferir nas condições nutricionais desse grupo. $\mathrm{O}$ presente trabalho objetivou avaliar o estado nutricional e o consumo alimentar dos estudantes ingressantes na Faculdade de Ciências Aplicadas (FCA) da UNICAMP, verificando a hipótese de que há um melhor padrão alimentar nos cursos da área da saúde em comparação a cursos de outras áreas. O estudo avaliou uma amostra de 351 alunos dos diversos cursos da instituição, por meio da coleta de medidas antropométricas: peso e altura, circunferência da cintura e quadril. Além disso, foi aplicado um questionário de frequência alimentar aos estudantes, que foi avaliado de acordo com o recomendado pela Pirâmide Alimentar Brasileira. Observou-se que há um baixo consumo de hortaliças e de frutas entre alunos ingressantes na FCA e, ao contrário do que se esperava com o presente trabalho, os alunos dos cursos da área da saúde, Ciências do Esporte e Nutrição, não apresentaram uma melhor adequação quanto ao consumo alimentar; contudo, apresentaram menor taxa de obesidade em comparação aos cursos de outras áreas, como Engenharia e Gestão.
\end{abstract}

Palavras-chave: avaliação nutricional, consumo alimentar, antropometria.

\section{Anthropometric assessment and analysis of dietary intake of university students in the Faculty of Applied Science - UNICAMP}

Besides teenagers' related conditions, changes associated with the beginning of university environment lifestyle, such as modifications in food intake, physical exercise, alcohol consumption and smoking, can influence both nutritional status and biological vulnerability of this group. This study aimed to evaluate the nutritional status and food intake of freshmen admitted to the School of Applied Sciences (SAS), UNICAMP. In addition, to verify the hypothesis that better results would be found among courses in the field of health compared to courses in other areas. In the study, a sample of 351 students was assessed by anthropometric measurements: weight, height, and waist-to-hip ratio. Also, a food frequency questionnaire was administered to the students in order to compare their consumption with the amount recommended by the Brazilian Food Pyramid. A low consumption of vegetables and fruits was observed among freshmen of SAS. On the contrary of that expected, students admitted to courses belonging to the field of health - Sport Sciences and Nutrition - did not show a better quality in food

${ }^{1}$ Faculdade de Ciências Aplicadas, Universidade Estadual de Campinas - UNICAMP. Correspondência: Rua Pedro Zacarias no 1.300, Jardim São Paulo, Limeira, SP. CEP 13484-350. Telefones: (11) 94810-1802 / (11) 3599-1919.E-mail: mayaralpm@gmail.com

${ }^{2}$ Docente da Faculdade de Ciências Aplicadas, Universidade Estadual de Campinas - UNICAMP. Correspondência: Rua Pedro Zacarias no 1.300, Jardim São Paulo, Limeira, SP. CEP: 13484350.E-mail:maykamiji@hotmail.com

${ }^{3}$ Docente da Faculdade de Ciências Aplicadas, Universidade Estadual de Campinas - UNICAMP. Correspondência: Rua Pedro Zacarias no 1.300, Jardim São Paulo, Limeira, SP. CEP: 13484 350.E-mail: torezzan@gmail.com

${ }^{4}$ Docente da Faculdade de Ciências Aplicadas, Universidade Estadual de Campinas - UNICAMP. Correspondência: Rua Pedro Zacarias no 1.300, Jardim São Paulo, Limeira, SP. CEP: 13484 350. E-mail:adriane.antunes@fca.unicamp.br 
intake; however, they did show a low percentage of obesity, compared to courses of other areas, such as Engineer and Management.

Key-words: nutritional assessment, dietary intake, anthropometry.

\section{INTRODUÇÃO}

A obesidade é definida como o excesso de acúmulo de gordura corporal e um fator de risco para várias doenças metabólicas, como diabetes, hiperlipidemia e câncer [1]. No Brasil, a obesidade já ultrapassou a desnutrição como doença mais prevalente em crianças e adolescentes [2].

Nas últimas décadas, houve um aumento, especialmente na população jovem, do consumo de alimentos ricos em "calorias vazias" (gêneros alimentícios com alto teor de açúcares e/ou gorduras e pobres em vitaminas e minerais), como fast-foods, refrigerantes e doces [3]. Esse tipo de dieta apresenta grandes concentrações de sódio, gorduras saturadas e trans, e de carboidratos refinados (consequentemente, pobres em fibras). O consumo de fast-food está fortemente associado com o ganho de peso e resistência à insulina, sugerindo que este tipo de alimento aumenta o risco de obesidade e de diabetes tipo $2^{[4-7]}$.

Jovens adultos que frequentam a faculdade demonstram uma propensão ainda maior de ganho de peso [8]. Somadas a situações próprias da adolescência, mudanças oriundas do ingresso no meio universitário, como modificações no padrão alimentar, na prática de atividade física, no estresse e no consumo de álcool e de cigarros podem interferir na formação física e social, bem como nas condições nutricionais e na vulnerabilidade biológica desse grupo ${ }^{[1,9]}$.

Segundo estudo de Brown et al [10], entre os fatores que mais influenciam a escolha alimentar dos estudantes estão o tempo limitado e a falta de dinheiro. Tais barreiras podem promover escolhas alimentares que incluem uma abundância de produtos processados, refeições pré-preparadas e fast-food, levando a uma monotonia alimentar. Outro fator relevante é a inexperiência dos estudantes na compra, no armazenamento e no preparo dos gêneros alimentícios, principalmente quando esses estudantes saem do âmbito familiar ${ }^{[11]}$.
Dado ao grande número de jovens adultos que frequentam a faculdade ou universidade a cada ano e as implicações para a saúde de ganho de peso nesta população, os primeiros anos de faculdade parecem ser um período crítico para a promoção do controle de peso. Além disso, alguns dos hábitos alimentares adquiridos nesse período estudantil continuam na idade adulta ${ }^{[8,11]}$.

$\mathrm{Na}$ literatura, são encontrados artigos que avaliaram a ingestão alimentar de estudantes de universidades [4,10,12-15]. Porém, até o presente momento, estudos que avaliaram o consumo alimentar de alunos pertencentes a cursos das três grandes áreas do conhecimento são escassos (Humanas, Biológicas e Exatas).

Assim, o objetivo do estudo foi avaliar o consumo alimentar de universitários para traçar um perfil alimentar de alunos ingressantes na Faculdade de Ciências Aplicadas/Unicamp (FCA), campus Limeira (São Paulo). Na sequencia, o estudo propôs-se a avaliar o estado nutricional e o consumo alimentar dos estudantes de todos os cursos da FCA, observando-se a ocorrência de melhores resultados de consumo alimentar e antropometria nos cursos da área da saúde.

\section{METODOLOGIA}

Foi realizado um estudo transversal durante o período de agosto de 2009 a julho de 2011. Na primeira etapa do estudo, objetivou-se traçar o perfil antropométrico e o hábito alimentar dos ingressantes na FCA. Para tanto, durante o período de agosto a dezembro de 2009, 191 alunos (grupo $\mathrm{A}=42 \%$ homens e $58 \%$ mulheres) foram selecionados aleatoriamente para participaram do estudo. Nesse período, foi elaborado e aplicado o questionário de Frequência Alimentar Qualitativo (QFA) [?]. Na segunda etapa, durante o período entre julho de 2010 a janeiro de 2011, outros 151 alunos (grupo $\mathrm{B}=42 \%$ homens e 58\% mulheres), representando todos os cursos da FCA, foram avaliados quanto à antropometria e ao consumo alimentar, além de serem entrevistados quanto à prática ou não de atividade 
física, e classificados como "ativos" quando houvesse a prática regular de exercícios no mínimo 3 vezes por semana, 30 minutos ou mais por dia [16]. Os voluntários eram pertencentes a cursos das três grandes áreas do conhecimento, Humanas (Gestão de Políticas Públicas, Gestão de Comércio Exterior, Gestão de Comércio Internacional e Gestão do Agronegócio), Biológicas (Ciências do Esporte e Nutrição) e Exatas (Engenharia de Manufatura e Engenharia de Produção). O projeto foi aprovado pelo Comitê de Ética em Pesquisa da Faculdade de Ciências Médicas da Unicamp (protocolo no 832/2009), e os alunos foram incluídos, mediante a assinatura de Termo de Consentimento Livre e Esclarecido.

Para avaliação dietética, foi empregado o QFA, [9. Para a análise dos dados obtidos, foi utilizado o Guia Adaptado para a População Brasileira [5] O consumo alimentar de cada aluno avaliado foi considerado "adequado" se estivesse dentro de 5 a 9 porções para cereais, 3 a 5 porções para frutas, 4 a 5 porções para hortaliças, 1 porção de leguminosas, 1 a 2 porções de carnes e ovos, 3 porções de leite e derivados, 1 a 2 porções de açúcares e doces e 1 a 2 porções de óleos e gorduras. Se o número de porções de cada grupo fosse maior ou menor que as faixas indicadas, o consumo alimentar foi considerado como "excessivo" ou "deficiente" respectivamente.

A avaliação antropométrica foi realizada pela determinação do Índice de Massa Corporal (IMC), que representa um indicador clínico reconhecido [17] além das medidas de circunferências (cintura e quadril) para cálculo da relação entre esses dois parâmetros (Relação Cintura/Quadril= RCQ). Os valores de referência utilizados para mulheres foram: <0,71(risco baixo), 0,71-0,8 (risco moderado) $>0,8$ (alto risco). E, para homens: $<0,8$ (risco baixo), 0,8-0,95 (risco moderado), $>0,95$ (alto risco) ${ }^{[17]}$.

Com os resultados obtidos, foi traçado um plano de intervenção nutricional que consistia em uma oficina que pretendeu transmitir aos alunos informações sobre preparações de alimentos saudáveis, de baixo custo e pequeno tempo para o preparo. $\mathrm{O}$ cardápio preparado incluía: carne com legumes, feijão, arroz, salada de agrião, tomate e cebola, banana de micro-ondas com canela de sobremesa e suco de laranja com talos de agrião. A oficina foi planejada em três etapas: panorama do consumo alimentar, elaboração de receitas, teste de aceitabilidade e teste de eficiência da oficina. Houve a entrega de um questionário para os participantes com vistas a avaliação a eficiência da oficina com as seguintes perguntas:

1- Você acredita que as deficiências nutricionais encontradas, entre os alunos da FCA foram abordadas de maneira clara e objetiva?

2- Foi possível compreender os conceitos realtivos à importância da alimentação equilibrada? Esses conceitos já faziam parte do seu conhecimento?

3- Você acredita que seja possível adicionar à sua dieta habitual as preparações apresentadas durante a oficina?

4- As preparações atenderam ao objetivo da oficina em relação ao custo e aos aspectos nutricionais?

Além disso, os alunos foram orientados a incluir sugestões e comentários. Houve a participação voluntária de 15 alunos, representantes de todos os cursos da FCA. Esses questionários foram elaborados pelos pesquisadores para avaliar a intervenção proposta.

Para a análise estatística, utilizou-se o método de Teste de Hipótese sobre a diferença entre proporções em duas populações, com nível de significância igual a 5\% $(p<0,05)$ no programa Microsoft Office Excel, versão 2010.

\section{RESULTADOS}

\section{Avaliação Dietética}

Ao traçar o perfil do consumo alimentar de todos os alunos da FCA, observou-se que grande parte dos alunos possui uma alimentação inadequada, que não se restringe a um grupo alimentar, e é mais evidente nos grupos de cereais, frutas e hortaliças. A população feminina do grupo A apresentou um elevado consumo de açúcares e doces, não observado na população masculina (Figura 1). 
Figura 1. Distribuição de alunos (ambos os sexos) dos cursos de Ciências do Esporte, Engenharia, Gestão e Nutrição, matriculados na Faculdade de Ciências Aplicadas (FCA), segundo adequação do consumo classificado de acordo com os grupos alimentares. Limeira, 2009.

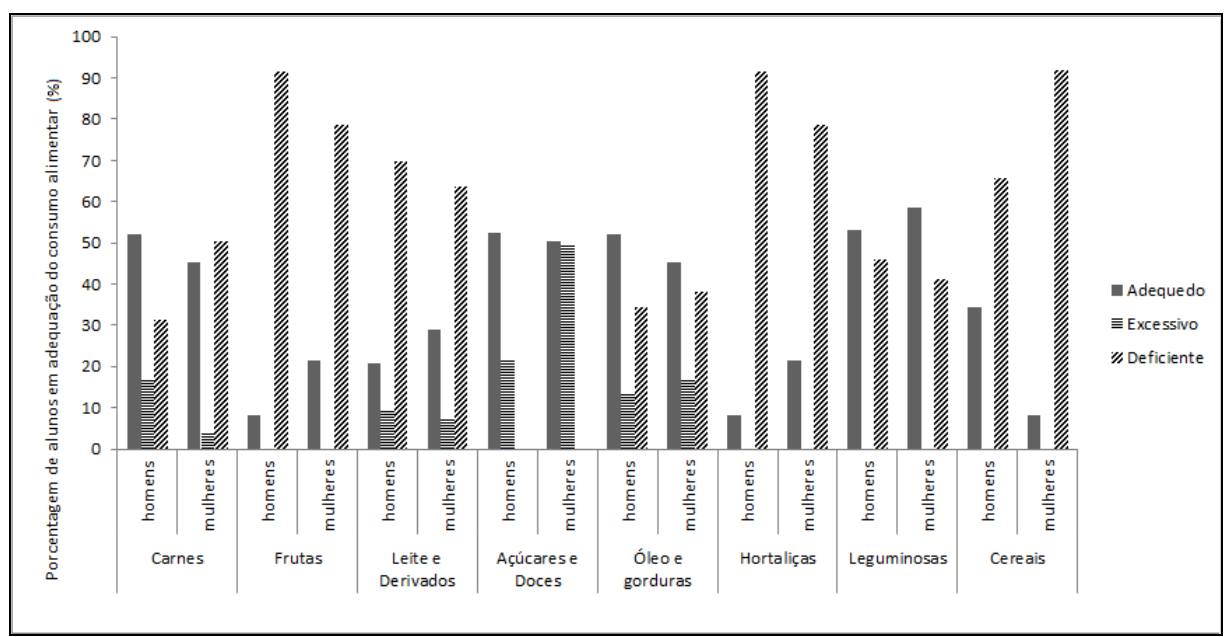

O perfil de consumo de cada um dos grupos de alimentos que compõe a Pirâmide Alimentar Brasileira foi semelhante entre os cursos de Ciência do Esporte e Nutrição, não sendo observada diferença estatisticamente significativa entre os resultados obtidos para os alunos dos dois cursos. Comparados aos demais cursos, porém, foram observadas diferenças em relação ao consumo (Figura 2).

Figura 2. Distribuição de alunos (ambos os sexos) dos cursos de Ciências do Esporte, Engenharia, Gestão e Nutrição, matriculados na Faculdade de Ciências Aplicadas (FCA), segundo adequação do consumo alimentar classificado de acordo com os grupos alimentares. Limeira, 2010-2011

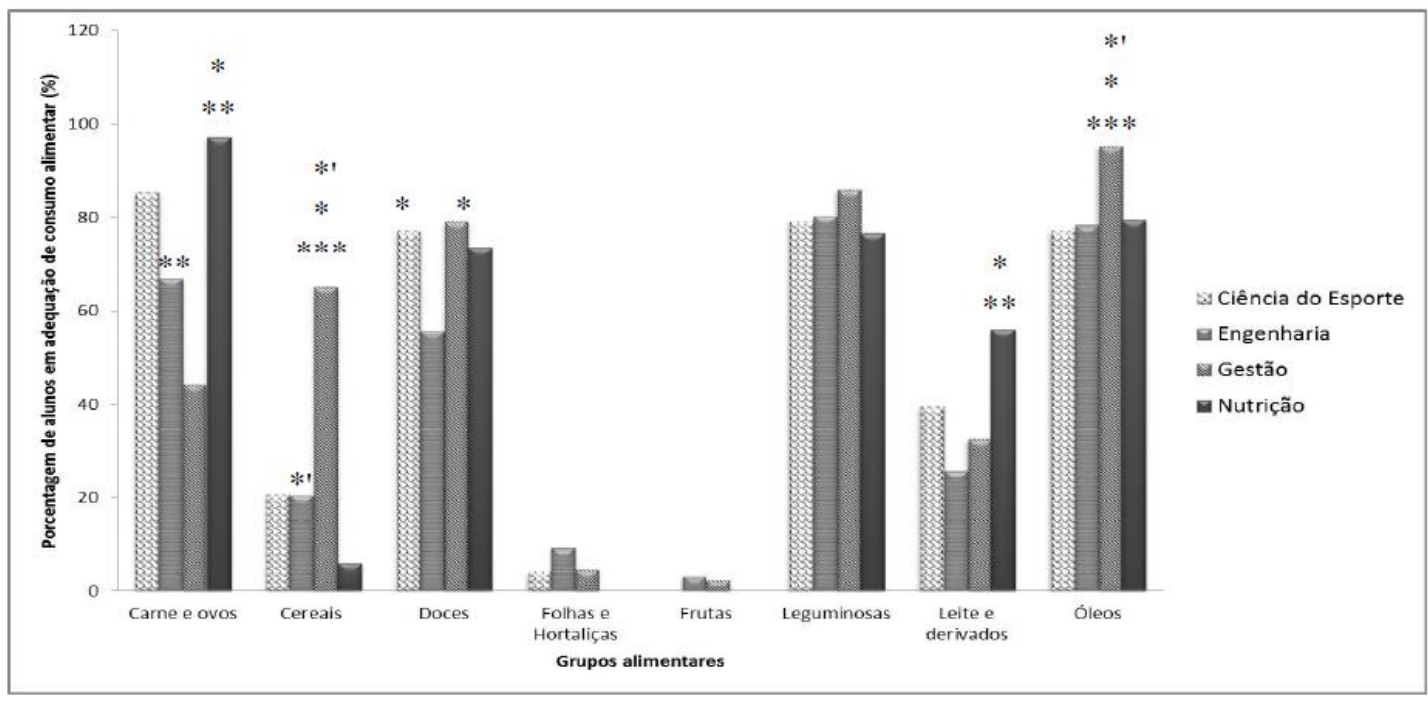

${ }^{*} p<0,05$ em comparação à Engenharia; ${ }^{* *} p<0,05$ em comparação Gestão; ${ }^{* * *} p<0,05$ em comparação à Ciências do Esporte; *’ $p<0,05$ em comparação à Nutrição, segundo Teste de Hipótese de duas populações. 


\section{Avaliação Antropométrica}

Por meio do cálculo do IMC foi possível observar que a maior parte da população do grupo A é classificada como eutrófica (Tabela 1). Porém, existe uma parcela importante de alunos do sexo masculino $(20 \%)$ identificados nos intervalos relativos a sobrepeso e obesidade (Tabela 1). Em relação ao IMC, observou-se um maior número de mulheres $(p<0,05)$ na faixa de baixo peso. Quanto à RCQ, a população feminina apresentou valores mais elevados em comparação aos valores da população masculina $(p<0,05)$.

Tabela 1. Perfil antropométrico dos alunos da Faculdade de Ciências Aplicadas (n=191). Limeira, 2009.

\begin{tabular}{c|cc}
\hline Estado Nutricional & \multicolumn{2}{|c}{ Sexo } \\
\cline { 2 - 3 } Intervalos de IMC & Mulheres & Homens \\
\hline$<18,5$ & $8(7 \%)^{*}$ & $0(0 \%)$ \\
$18,5-24,99$ & $91(83 \%)$ & $60(75 \%)$ \\
$25,5-29,99$ & $8(7 \%)^{*}$ & $14(18 \%)$ \\
$>30$ & $4(4 \%)$ & $6 \quad(8 \%)$ \\
& & \\
Relação Cintura/Quadril & & \\
Baixo Risco & $51(46 \%)$ & $41(51 \%)$ \\
Risco Moderado & $39(35 \%)$ & $34(43 \%)$ \\
Alto Risco & $21(19 \%)^{*}$ & $5(6 \%)$ \\
\hline${ }^{*}<0,05$ em comparaçao a população masculina. & &
\end{tabular}

De maneira semelhante, os alunos do grupo B foram classificados, em sua maioria, como eutróficos. Porém, os cursos de Engenharia e de
Gestão possuem a maior parcela de estudantes classificados como obesos $(p<0,05)$ (Tabela 2).

Tabela 2. Estado nutricional, de acordo com intervalos do Índice de massa corporal (IMC) dos alunos da FCA $(n=151)$. Limeira, 2011.

\begin{tabular}{cccccc}
\hline Intervalos de IMC & Ciência do Esporte & Cursos Engenharia & Gestão & Nutrição \\
\hline$<18,5$ & $6(13 \%)$ & $0^{*}$ & $3(7 \%)$ & $3(10 \%)$ \\
$18,5-24,99$ & $32(70 \%)$ & $27(87 \%)$ & $3(75 \%)$ & $24(80 \%)$ \\
$25-29,99$ & $7(15 \%)$ & $3(7 \%)$ & $7(16 \%)$ & $1(10 \%)$ \\
$>30$ & $1(2 \%)$ & $12(29 \%)^{* * * *}$ & $13(30 \%)^{* * * *}$ & 0 \\
Total & 46 & 42 & 26 & 30 \\
\hline
\end{tabular}

${ }^{*} p<0,05$ em comparação a Ciências do Esporte ${ }^{* *} p<0,05$ em comparação à Nutrição, segundo Teste de Hipótese de duas populações.

Quanto à RCQ, os alunos de Ciências do Esporte apresentaram a maior parte da população com baixa RCQ $(p<0,05)$ (Tabela 3). 
Tabela 3. Relação Cintura/Quadril dos alunos da Faculdade de Ciências Aplicadas, de acordo com o sexo. Limeira, 2011.

\begin{tabular}{|c|c|c|c|c|c|c|}
\hline \multirow{2}{*}{\multicolumn{2}{|c|}{ Relação cintura/quadril/sexo }} & \multicolumn{5}{|c|}{ Cursos } \\
\hline & & & \multirow{2}{*}{$\begin{array}{c}\begin{array}{c}\text { Ciências do } \\
\text { Esporte }\end{array} \\
1(2 \%)\end{array}$} & \multirow{2}{*}{$\begin{array}{c}\text { Engenharia } \\
3(10 \%)\end{array}$} & \multirow{2}{*}{$\begin{array}{c}\text { Gestão } \\
5(11 \%)\end{array}$} & \multirow{2}{*}{$\begin{array}{l}\text { Nutrição } \\
4(13 \%)\end{array}$} \\
\hline Sexo Feminino & Baixo risco & $<0,73$ & & & & \\
\hline & Moderado & 0,73 a 0,8 & $9(19 \%)$ & $9(29 \%)$ & $12(27 \%)$ & $21(70 \%)$ \\
\hline & Alto risco & $>0,8$ & $4(9 \%)$ & $3(10 \%)$ & $12(27 \%)$ & $5(17 \%)$ \\
\hline Total $(n=88)$ & & & 14 & 15 & 29 & 30 \\
\hline \multirow[t]{3}{*}{ Sexo Masculino } & Baixo risco & $<0,88$ & $29(63 \%)$ & $15(48 \%)$ & $11(25 \%)$ & $0(0 \%)$ \\
\hline & Moderado & 0,88 a 0,95 & $2(4 \%)$ & $1(3 \%)$ & $4(9 \%)$ & $0(0 \%)$ \\
\hline & Alto risco & $>0,95$ & $1(2 \%)$ & $0(0 \%)$ & $0(0 \%)$ & $0(0 \%)$ \\
\hline Total $(n=63)$ & & & 32 & 16 & 15 & 0 \\
\hline \multirow[t]{4}{*}{ Total $(n=151)$} & Baixo risco & & $30(65 \%)^{* * * *}$ & $18(58 \%)^{* *}$ & $16(36 \%)$ & $4(13 \%)$ \\
\hline & Moderado & & $11(24 \%)^{* *}$ & $10(32 \%)$ & $16(36 \%)$ & $21(70 \%)^{*, * *+}$ \\
\hline & Alto risco & & $5(11 \%)$ & $3(10 \%)$ & $12(27 \%)$ & $5(17 \%)^{*}$ \\
\hline & & & $46(30 \%)$ & $31(21 \%)$ & $44(29 \%)$ & $30(20 \%)$ \\
\hline
\end{tabular}

†A amostra de Nutrição era composta essencialmente de mulheres.

$* p<0,05$ em comparação a Gestão; ** $p<0,05$ em comparação à Nutrição, *** $p<0,05$ em comparação á Engenharia segundo Teste de Hipótese de duas populações.

Os números entre colchetes são os percentuais em relação ao total $(\mathrm{n}=151)$ da amostra.

Quanto à prática de atividade física, 20\% dos alunos de Ciências do Esporte, 12\% dos alunos de Nutrição, $32 \%$ dos alunos de Engenharia ( $p<0,05 \mathrm{em}$ comparação à Nutrição), e 45\% dos alunos de Gestão $(p<0,05$ em comparação aos demais cursos) relataram adotar a referida prática.

\section{Intervenção nutricional}

A oficina contou com a participação voluntária de 15 alunos, sendo uma aluna de Engenharia de manufatura, um aluno de Gestão de Políticas Públicas, duas alunas de ciências do esporte e onze alunas de nutrição. Após a explicação inicial sobre o panorama do consumo alimentar e antropométrico dos alunos da FCA, os alunos foram orientados no preparo de receitas e realizaram um teste sensorial no qual os alunos analisaram a textura, odor e aceitabilidade e avaliaram a eficiência da oficina. A aceitabilidade das receitas foi boa e, dos 15 participantes, 14 avaliaram a oficina como eficiente e afirmaram que as preparações poderiam ser inclusas na alimentação diária. Um dos participantes relatou que a oficina foi boa, mas que ainda sim teria dificuldades em implementar algumas das preparações devido as atividades da faculdade.

\section{DISCUSSÃO}

No presente trabalho, avaliamos o perfil nutricional dos alunos de diferentes cursos da FCA. Observamos uma alimentação qualitativamente deficitária e um número expressivo de alunos identificados com sobrepeso e obesidade. A proporção desses distúrbios revelou associação (positiva) com alguns cursos.

Por meio da avaliação dietética, observamos um consumo deficiente de cereais, de frutas e de hortaliças entre os alunos de todos os cursos. A grande inadequação dos grupos de frutas e de hortaliças encontrada nesse trabalho corrobora como o estudo de Racette et al. ${ }^{[18]}$, que revelou que $70 \%$ da população de estudantes comiam menos de 5 frutas e legumes diariamente.

O consumo desses alimentos tem sido recomendado a fim de diminuir a incidência de diversas doenças crônicas. Entretanto, autores têm destacado que seu consumo está abaixo da recomendação mundial em diversos países [19]. Além disso, sabe-se que o consumo de cereais integrais, frutas e hortaliças está associado a um aumento no teor de fibras da alimentação, que atuam na redução do apetite, no aumento da saciedade e no retardo da absorção dos carboidratos após as refeições [20]. 
Melby et al ${ }^{[14]}$ avaliaram o consumo alimentar dos alunos que viviam dentro e fora do campus universitário. Os autores relataram que, apesar do conhecimento sobre a importância do consumo adequado de hortaliças e frutas, os estudantes possuem uma baixa ingestão desses alimentos, principalmente devido ao custo e a inconveniência das compras dos gêneros alimentícios. Vieira et al [15] constataram, em seu estudo, que os adolescentes recém ingressos na universidade consumiam quatro vezes menos frutas que a quantidade recomendada.

Além da deficiência no consumo de frutas e de hortaliças, no presente estudo, observou-se um maior consumo de doces pelas alunas do grupo A, que contribui ainda mais para a deficiência de vitaminas e minerais.

Driskell et al. [21], em um amplo estudo sobre as diferenças entre os sexos em relação ao consumo de fast-food, observaram que a população feminina é mais propensa para pular refeições como meio de controle de peso, é mais susceptível a influências de informações nutricionais na escolha dos pratos, e possui percepção do peso corporal mais distorcida que a masculina. Georgiou et al. [4] avaliaram o consumo alimentar de jovens adultos (18 a 24 anos de idade), estudantes e não estudantes universitários, e encontraram um baixo consumo de hortaliças em mulheres, independentemente do status educacional. Além disso, há uma possível associação entre variações de paladar, com predileção a doces e alimentos com maior teor de gordura, e fases do ciclo menstrual [20].

Observou-se ainda um número importante de alunos com sobrepeso/obesidade, possivelmente consequência de uma alimentação inadequada. A prática de atividade física foi relatada por $59 \%$ dos alunos e, diferentemente do que se encontrou no estudo de Marcondelli et al. [22], tal prática foi identificada entre uma reduzida proporção $(20 \%)$ dos alunos do curso de Ciências do Esporte.

Lloyd-Richardson et al. [23] avaliaram 904 alunos ingressantes em uma universidade pública norte-americana, observando um ganho de peso médio de $3,5 \mathrm{~kg}$ tanto em homens como em mulheres. Lowe et al. ${ }^{[24]}$ consideram o primeiro ano de faculdade como um período de risco aumentado para ganho de peso, uma vez que, nesse período, ocorrem aumentos significativos no peso corporal, no IMC e na porcentagem de massa de gordura. Além disso, Raccete et al. ${ }^{[18]}$ demonstraram um aumento do peso corporal e do IMC dos alunos durante os anos da faculdade. Tais dados ressaltam a relevância de atividades educacionais em nutrição, a fim de se prevenir que os números de sobrepeso/obesidade se tornem ainda mais expressivos.

A oficina realizada na FCA pode ser um exemplo de intervenção nutricional já que obteve grande aceitação pelos alunos participantes, entretanto outras estratégias de intervenções nutricionais que visam informar e reeducar um maior número de alunos devem ser desenvolvidas.

O presente estudo demonstrou que alunos ingressantes em todos os cursos da Faculdade de Ciências adotam uma alimentação inadequada, que não se restringe a um grupo alimentar, e é mais evidente nos grupos de cereais, frutas e hortaliças. Ao contrário do que se esperava com o presente trabalho, alunos dos cursos da área da saúde, Ciências do Esporte e Nutrição, não apresentaram uma melhor adequação quanto ao consumo alimentar; contudo, apresentaram menor taxa de obesidade em comparação aos cursos de outras áreas, como Engenharia e Gestão.

\section{AGRADECIMENTO}

Agradecemos ao CNPq pelo apoio financeiro, na forma de bolsa, para a primeira autora.

\section{REFERÊNCIAS}

[1] Bu SY. Transitional changes in energy intake, skeletal muscle content and nutritional behavior in college students during course-work based nutrition education. Clin Nutr Res. 2013;2(2):125-34.

[2] Duncan S, Duncan EK, Fernandes RA, Buonani C, Bastos KD, Segatto AF, et al. Modifiable risk factors for overweight and obesity in children and adolescents from Sao Paulo, Brazil. BMC Public Health. 2011;11(585):1-9.

[3] Ebbeling CB, Pawlak DB, Ludwig DS. Childhood obesity: public-health crisis, common sense cure. Lancet. 2002;360(9331):473-82.

[4] Georgiou CC, Betts NM, Hoerr SL, Keim K, Peters PK, Stewart B, et al. Among young adults, college students and graduates practiced more healthful habits and made more healthful food choices than did nonstudents. J Am Diet Assoc. 1997;97(7):754-9. 
[5] Philippi ST, Latterza AR, Cruz ATR, Ribeiro LC. Pirâmide alimentar adaptada: guia para escolha dos alimentos. Rev Nutr. 1999;12(1):65-80.

[6] Ramalho RA, Saunders C. O papel da educação nutricional no combate às carências nutricionais. Rev Nutr. 2000;13(1):11-6.

[7] Stanner S. Nutrition and teenagers. Women's Health Med. 2004;1(1):6-10.

[8] Dennis EA, Potter KL, Estabrooks PA, Davy BM. Weight gain prevention for college freshmen: comparing two social cognitive theory-based interventions with and without explicit self-regulation training. J Obes. 2012;2012(2012):[10 p.].

[9] Ribeiro AC, Sávio KEO, Rodrigues MLCF, Costa THM, Schmitz BAS. Validação de um questionário de freqüência de consumo alimentar para população adulta. Rev Nutr. 2006;19(5):553-62.

[10] Brown L, Rosner B, Willett WW, Sacks FM. Cholesterol-lowering effects of dietary fiber: a meta-analysis. Am J Clin Nutr. 1999;69(1):30-42.

[11] Haberman S, Luffey D. Weighing in college students' diet and exercise behaviors. J Am Coll Health Health. 1998;46(4):189-91.

[12] Brevard PB, Ricketts CD. Residence of college students affects dietary intake, physical activity, and serum lipid levels. J Am Diet Assoc. 1996;96(1):35-8.

[13] Marlett JA, McBurney MI, Slavin JL. Position of the American Dietetic Association: health implications of dietary fiber. J Am Diet Assoc. 2002;102(7):993-1000.

[14] Melby CL, Femea PL, Sciacca JP. Reported dietary and exercise behaviors, beliefs and knowledge among university undergraduates. Nutr Res. 1986;6(7):799-808.

[15] Vieira VCR, Priore SE, Ribeiro SMR, Franceschini SCC, Almeida LP. Perfil socioeconômico, nutricional e de saúde de adolescentes recém-ingressos em uma universidade pública brasileira. Rev Nutr. 2002;15(3):273-82.
[16] Matsudo SM, Matsudo VR, Araújo T, Andrade D, Andrade E, Oliveira L, et al. Nível de atividade física da população do Estado de São Paulo: análise de acordo com o gênero, idade, nível socioeconômico, distribuição geográfica e de conhecimento. Rev Bras Cien Mov. 2002;10(4):41-50.

[17] World Health Organization. Obesity: preventing and managing the global epidemic: report of a WHO consultation. Geneva: World Health Organization; 2000. 252 p. (World Health Organization technical report series; 894.

[18] Racette SB, Deusinger SS, Strube MJ, Highstein GR, Deusinger RH. Weight changes, exercise, and dietary patterns during freshman and sophomore years of college. J Am Coll Health. 2005;53(6):245-51.

[19] Alves HJ, Boog MCF. Representações sobre o consumo de frutas, verduras e legumes entre fruticultores de zona rural. Rev Nutr. 2008;21(6):705-15.

[20] Frye CA, Crystal S, Ward KD, Kanarek RB. Menstrual cycle and dietary restraint influence taste preferences in young women. Physiol Behav. 1994;55(3):561-7.

[21] Driskell JA, Meckna BR, Scales NE. Differences exist in the eating habits of university men and women at fast-food restaurants. Nutr Res. 2006;26(10):524-30.

[22] Marcondelli PC, Costa THM, Schmitz BAS. Nível de atividade física e hábitos alimentares de universitários do $3^{\circ}$ ao $5^{\circ}$ semestres da área da saúde. Rev Nutr. 2008;21(1):3947.

[23] Lloyd-Richardson EE, Bailey S, Fava JL, Wing R. A prospective study of weight gain during the college freshman and sophomore years. Prev Med. 2009;48(3):25661.

[24] Lowe MR, Annunziato RA, Markowitz JT, Didie E, Bellace DL, Riddell L, et al. Multiple types of dieting prospectively predict weight gain during the freshman year of college. Appetite. 2006;47(1):83-90. 